\title{
Age dynamics of cerebral blood flow velocity in orthopedic diseases and fractures of limb bones
}

\begin{abstract}
Aim: Work done for the purpose of analysis of the dependence of the pace of age-related decline in speed of cerebral blood flow and functional status of the musculoskeletal system of different groups of patients.

Technique: Investigated the blood flow velocity (FV) in middle cerebral arteries (MCA) during the function of muscle samples of healthy people, as well as for fractures and diseases of the extremities (105 people), as well as congenital diseases with impaired growth of the extremities (71) in the treatment by Ilizarov.

Results: Based on constructing the equations of linear regression evaluation of age-related decline in the values of FV up to $60 \%$ of the constant component revealed that the source healthy people, the prognosis of life expectancy close to the regional norma (69-73 years). At the same time, age-related decline of FV in the MCA is a necessary condition for the preservation of cerebral autoregulation. The case of congenital pathology of the original $\mathrm{FV}$ in the MCA below, and the decrease rate is higher. The output of scope change FV in functional tests within the normal range $(25 \%)$ is accompanied by decreased capacity of patients and slowing down the process of reparative regeneration.
\end{abstract}

Volume 5 Issue $6-2018$

\author{
Schurov VA, Novicov KI, Muradisinov SO \\ Ilizarov Research Center for Rehabilitating Traumatology and \\ Orthopedics, Kurgan, Russia
}

\begin{abstract}
Correspondence: Schurov VA, Doctor of medical Sciences, Professor, Chief scientific, llizarov Research Center for Rehabilitating Traumatology and Orthopedics, Kurgan, Russia, Tel
\end{abstract} +7919 571 4942, Email shchurowland@mail.ru

Received: September 28, 2018 | Published: November 15, 2018

Keywords: cerebral blood flow, middle cerebral artery, age, pathology of the musculoskeletal system

\section{Introduction}

With increasing age comes the slowing of blood flow through the arteries of the brain. ${ }^{1-5}$ It is shown that at the same time, the elderly (419) was a slowdown in the speed of locomotion. ${ }^{6}$ When the pathology of the musculoskeletal system age-related decline rate of cerebral blood flow velocity are relatively higher. ${ }^{7,8}$ With increasing age, decreased as the maximum permissible values of the operational elongation according to the Ilizarov method stunting of the limbs and the length of the period of fixing broken bones. ${ }^{9,10}$

The aim of this work was the analysis of dependence of the pace of age-related decline in speed of cerebral blood flow and functional status of the musculoskeletal system of different groups of patients.

\section{Material and methods}

The object of the study was 61 patient aged 5 to 62 years with a lag in growth of one lower limb on the values from 2 to $15 \mathrm{~cm}$ due to the longitudinal ectromelia and 25 patients with fosfat-diabetes. The studies were performed before, during rapid elongation of the limb segments according to the Ilizarov method and after it. Also surveyed a group of 30 patients with gonarthrosis stage 1-3 (18-72 years), 30 patients with closed spiral fractures of the tibia (18-65 years is in the process of treatment according to Ilizarov).

The Blood flow velocity (FV) in middle cerebral arteries (MCA) were determined using ultrasonic installation "Angiodin 2 UK" (Russia) with sensors with carrier frequency as $2 \mathrm{MHz}$. Check FV for the MCA from each side repeating the functional test with compression of the elastic expander brush right and then left hand. It was determined the maximal amplitude of changes decreased systolic SK from the original values of each of the sides of the brain.

Self-esteem physical and mental state of patients was performed using test SF-36 incorporated in the computer test card contains 36 multiple-choice questions that are based on the 8 modules allows to judge about the quality of life by integrating data on the physical (availability, ability to self-care, well-being, feeling pain) and mental state (mood, mood, emotional state, social activity) of the patient. Evaluate each of the end modules is an indicator that ranges from 0 to 100 points.

Statistical processing of research results was conducted using analysis ToolPak "Microsoft Exell-2010. Given average values and standard error $(\mathrm{M} \pm \mathrm{m})$. To assess the statistical significance of differences of results of analysis of two independent samples was used t-Student test.

\section{Results}

If we trace the dynamics of the age-related decline in FV in the different study groups, we can construct the corresponding graphs and to determine when FV on the MCA will be reduced by $60 \%$ from the level of the DC component. This decrease in norm occurs at the approach of the indicator to the magnitude of the life expectancy of the population of the region and reflects the exhaustion of functional reserves of adaptation of the centres of the nervous system.

By reducing $\mathrm{FV}$ of MCA is $60 \%$, for example, in patients with gonarthrosis, the patients capacity drops from $75 \%$ to $15 \%$ (Figure 1).

The value of FV in the MCA in healthy children is $120 \pm 4.5 \mathrm{~cm} / \mathrm{s}$, It is more than patients with fractures and in children with congenital diseases of the limbs, but lower than in patients with gonarthrosis. When plotting the age dynamics of this indicator it is possible to define a threshold reduction of $60 \%$ and the number of years during which will be achieved such a reduction.

To calculate life expectancy based on the formula: $T=B * 0,4 / A$, where - constant component of the equation of linear regression, A the angular coefficient of the equation. The average life expectancy of 
men and women in the Kurgan region is 62 и73. In healthy people, a critical reduction of FV in the MCA will be achieved through 69 years in patients with gonarthrosis stages 1-3 in 72 years (mostly women), in patients with fractures of Shin bones through 69 years (Figure 2). The decline (the constant component of the equation of the linear regression) during treatment did not lead to the reduction of the duration of life of patients.

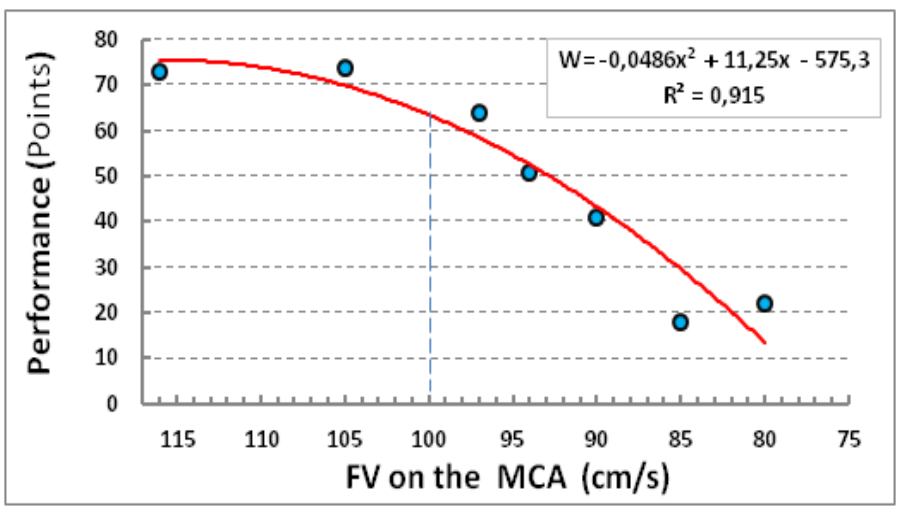

Figure I The dependence of the capacity of patients with gonarthrosis of the value of $\mathrm{FV}$ in the MCA.

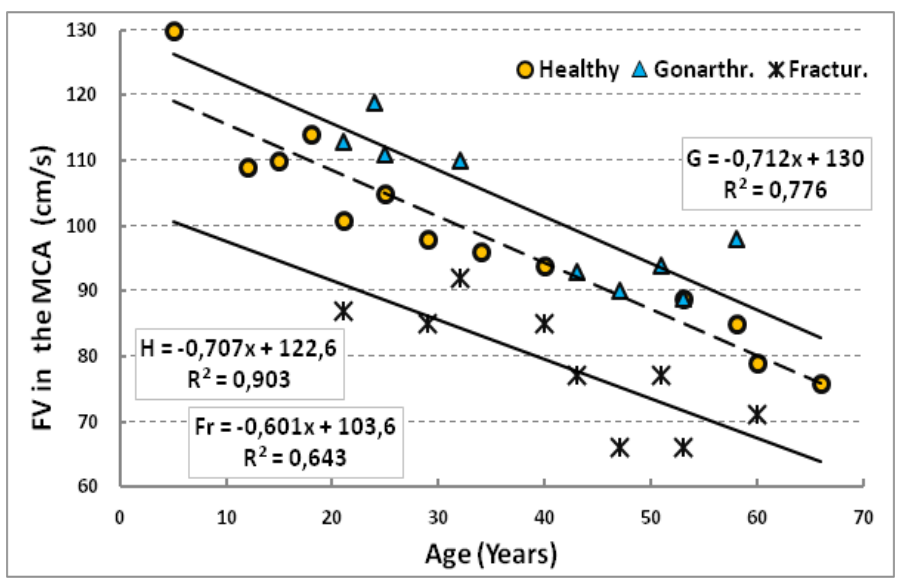

Figure 2 Age dynamics of blood flow velocity in the MCA in healthy and patients with osteoarthritis, and fractures of the Tibia bones.

Patients with congenital shortening of lower limbs, the constant component of the age-related decline in the value of $\mathrm{FV}$ is described by the equation $\mathrm{Sh}=0,649 \mathrm{x}+102,54$; $(\mathrm{R} 2=0,937)$. We found no significant differences in the dynamics of this indicator in patients with longitudinal ectromelia and phosphate diabetes. On the chart of their data is presented in one General group. In this the constant component is reduced compared with a norm of $16.4 \%$, and the slope is $7.2 \%$. Critical value of the reduction indicator will be achieved in 63 years. Thus in patients older than 40 years of absolute values of FV to MCA is lower than the critical values in healthy. Patients the sample is degenerate in nature and more appropriately the age dynamics of the ratio of FV to describe not linear, and power function (Figure 3).

Age-related decline of the FV in the arteries of the brain occurred against the background of maintaining the magnitude of changes in FV when conducting functional muscle loads, which allowed to judge the state of cerebral autoregulation. The increase in FV in the stress tests was greater, the less was the source of the absolute values of this parameter: $\Delta \mathrm{V}=0,174-0,0018 \times \mathrm{V} ; \mathrm{r}=-0,773$. Therefore, despite the age-related decline in speed of cerebral blood flow, reactivity indices of cerebral blood flow when performing muscle function tests are not violated. ${ }^{11}$

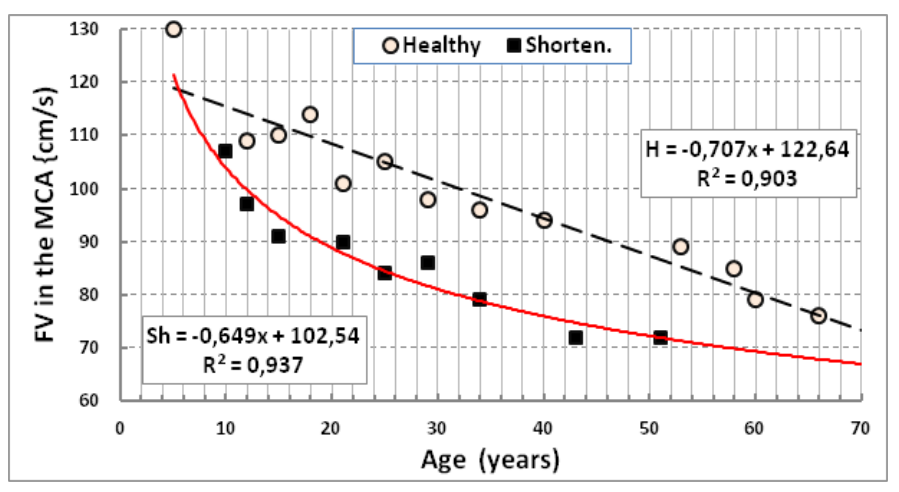

Figure 3 Age dynamics of FV to CMA in normal individuals and in patients with congenital limb shortening.

Of violation of cerebral autoregulation can be measured by the increase in the amplitude changes of FV in the MCA in functional tests with the muscles of the wrist grab. The value of this scale was determined largely by the depth of decline was not supposed to go beyond $25 \%$. The threshold value is exceeded, evidence of impaired regulation of cerebral hemodynamics and Central nervous system functions. When the breach was slowing down the process of reparative regeneration of the bone. The output indicator of the scope of changes permissible limits occurs in the treatment of fractures of tibia the lower values of FV in patients older than 50 years (Figure 4).

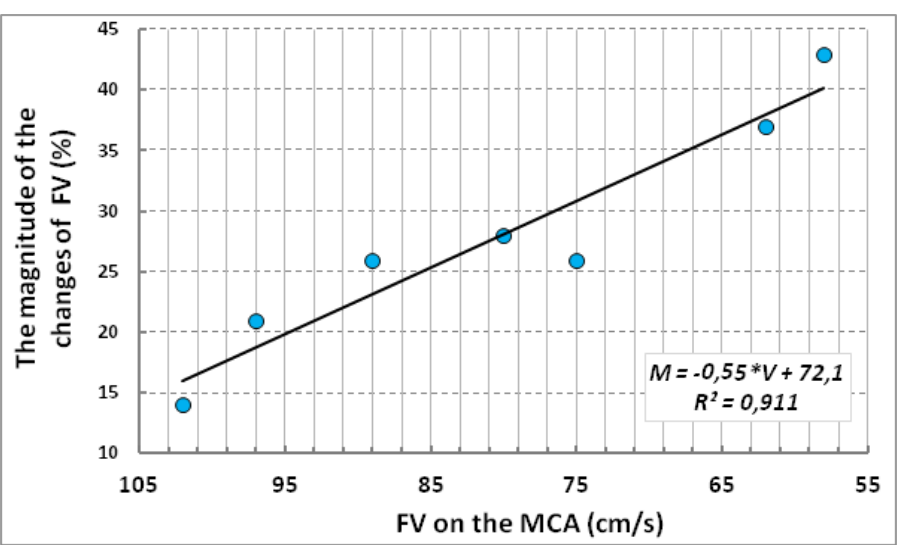

Figure 4 The dependence of the scale change on the magnitude of FV in patients with fractures of the tibia in the treatment process.

The decrease in FV in the MCA is characterized not only with increasing age of patients and severity of pathology (Figure 5). The larger the gap one of the limbs in longitudinal size, the lower FV on the contralateral side of the brain.

Even faster with the increasing age of patients decreased the indicators of FV in the MCA in surgical lengthening of the affected leg. In the treatment of patients with congenital limb shortening ratio of the values of FV to MCA on the contralateral and ipsilateral sides of the brain decreased to $74 \%$ (Figure 6).

During this period, the treatment of patients scope changes FV in MCA in the implementation of samples with the muscles of the wrist 
grab was increased in comparison with the quantities of changes of healthy people, In the process of the distraction scale was increased to $33 \%$. By the end of the distraction indicator of the scope has stabilized at $22 \% \pm 1.6$ (Figure 7 ), characteristic for healthy people.

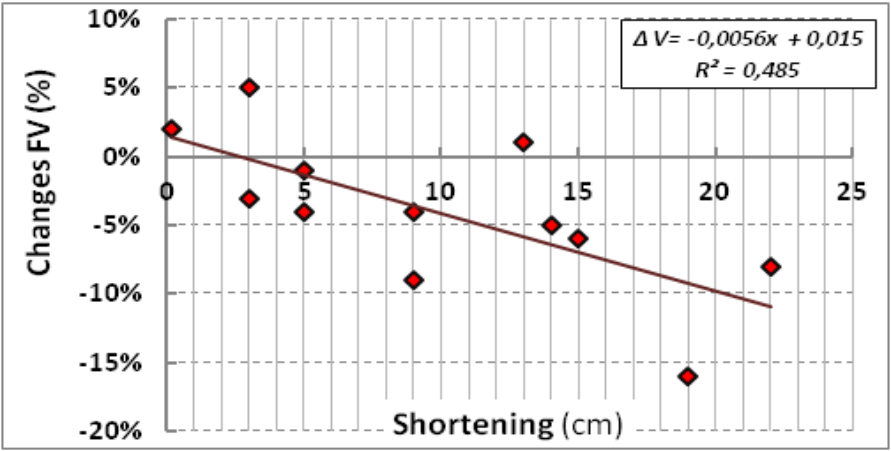

Figure $\mathbf{5}$ The dependence of FV on the MCA contralateral side when holding the muscle samples from the magnitude of limb shortening.

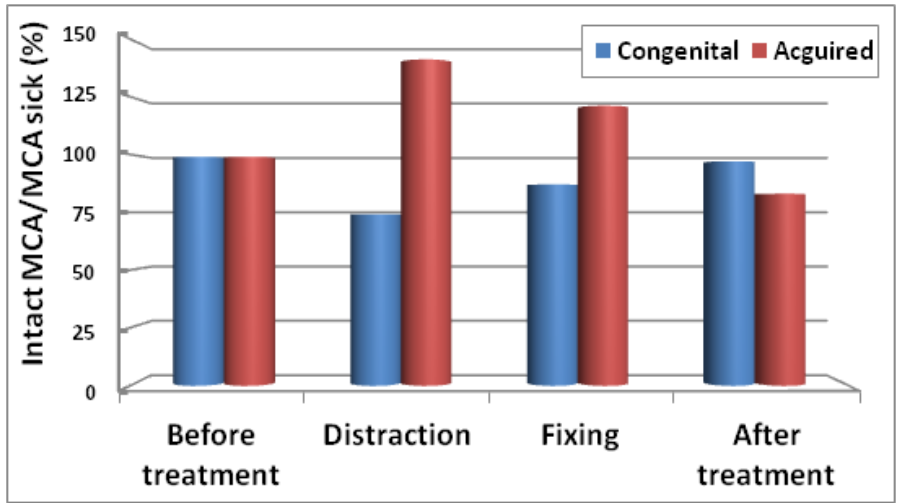

Figure 6 The dynamics of the ratio of FV in the MCA on the ipsilateral and contralateral sides of the brain in surgical leg lengthening.

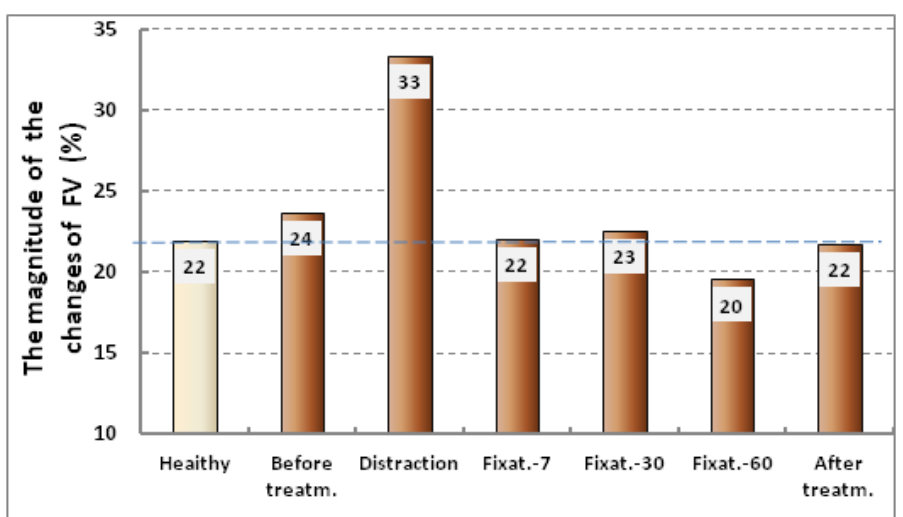

Figure 7 The dynamics of the magnitude of the changes on FV to MCA in the process of surgical treatment of patients with congenital shortening of the limb.

The health of the patients during the period of distraction was broken. The preservation of the variability of the magnitude of changes in SC within the corridor standards is a necessary condition for maintaining the normal functioning of the centers of the brain. During the test, SF-36 revealed that the more limiting effect of pain on daily activities of patients during distraction (P, points), the lower was the value of $\mathrm{FV}$ in the MCA contralateral side: $\mathrm{Vc}=0,52 * \mathrm{P}+69$; $(r=0,425)$. The smaller was the relative value of $\mathrm{FV}$, the lower the capacity of patients (Figure 8).

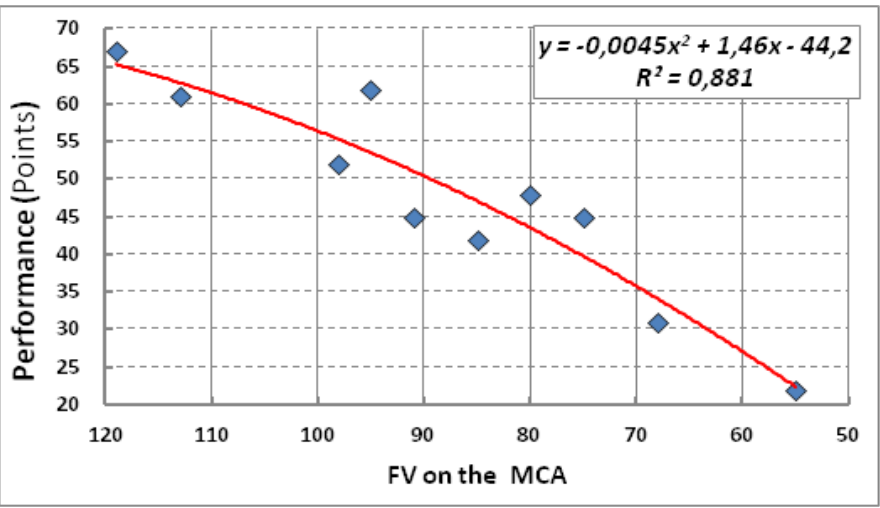

Figure 8 The relationship between the capacity of patients and relative FV in the MCA contralateral side of the brain during surgical lengthening of the limb.

\section{Discussion}

Literature data on age-related decline in FV in the MCA were mostly obtained during examination of healthy people and patients with arterial hypertension, ${ }^{3,12}$ including the children's people ${ }^{2,5}$ and elderly. ${ }^{4}$ The reason for the decline of FV researchers was seen in age-related reduction in minute volume of the heart, ${ }^{13}$ the reduced metabolism of the nervous centres and the sensitivity of chemoreceptors to carbon dioxide. ${ }^{5,7}$ In our study confirmed that with increasing age of FV to MCA really reduced in healthy humans, and orthopedic pathology. However, it is shown that the constant component of the regression equation above the norm in diseases of the joints and below the surgical treatment by Ilizarov diseases of the limbs and bone fractures. Existing hypotheses of decline do not allow to explain the nature of such differences.

Age-related decline of FV to MCA so naturally, that allows for probabilistic projections of life expectancy. The figure is not so much the original values of SK to AGR, as the pace of decline, the Prognosis worsens with systemic congenital diseases. In such patients the initial high performance of the IC to 10-15 years become significantly lower than in healthy peers. After 30 years the pace of further decline is slowing. When conducting functional muscle samples shows that the implementation of the mechanism of cerebral autoregulation, it is necessary to decrease absolute values of FV to MCA. This reduction is observed not only with increasing age but also in the treatment of patients with bone fractures, patients with congenital disorder of longitudinal growth of the limb.

The heavier orthopedic pathology, the greater the tendency to reduce the speed of cerebral blood flow during the load functional tests. For example, during distraction FV in the MCA contralateral side of the brain falls below $25 \%$. During this period, the magnitude of the changes of the index beyond the boundaries of tolerance. In the period of the surgical treatment of disturbed health of patients. Therefore, patients with bone fractures, especially in the elderly need to be sparing motor mode, a longer stay in stationary conditions, because the reduction in age-related reactivity in patients significantly increases the duration of the fixation period. ${ }^{9,14,15}$ 


\section{Summary}

A. Age-related decline of FV to MCA is a natural phenomenon, since it contributes to the preservation of cerebral autoregulation.

B. The relatively low absolute performance in the FV in the MCA and increased the rate of their age-related decline are the result of violations of patients " health and determine the worst prognosis of life expectancy.

C. The output of scope change FV in the MCA beyond the norm in the surgical treatment of patients accompanied by a decrease of their capacity and slow down the process of reparative regeneration.

\section{Acknowledgements}

None.

\section{Conflict of interest}

The authors declare no conflict of interest.

\section{References}

1. Andreyev Yu, Weinstein GB, Semeriya VN. Studies of changes in cerebral blood flow and cerebrovascular reactivity during the late postnatal ontogeny. J Sib Evolutional Biochem and Physiology. 2013;49(6):457-459.

2. Bode H. Pediatrics application transcranial Doppler sonography. New York, Vienna: Springer Verlag; 1988:108.

3. Lelyuk VG, lelyuk SE. Ultrasonic Angiology M. 2003:324.

4. Rautenberg W. Transcranialle Doppler sonographie. Dureblutengsstorunden des gehirnsneue diagnostike Moglichkeiten. Dusseldorf. 1987:30-55.

5. Vatolin KV. Ultrasound diagnosis of brain diseases in children. Vidar. 2000;2:96-112.
6. Sorond FA, Galica A, Serrador JM, et al. Cerebrovascular hemodynamics, gait, and falls in an elderly population mobilize Boston Study. Neurology. 2010;74(20):627-1633.

7. Schurov VA. Analysis of the mechanism of the decrease in the rate of cerebral blood flow. Regional circulation and microcirculation. 2016;2(58):4-50.

8. Schurov VA. Autoregulation of cerebral hemodynamics in the clinic of traumatology and orthopedics. Moskau. 2017:114.

9. Schurov IV. Chronobiological, socio-economic and biological factors that determine the regenerative ability of the bone. Scientific Bulletin of the Khanty-Mansiysk state medical Institute. 2006;1:134-135.

10. Schurov VA, Muradisinov SO. The second rule of regeneration and leg elongation of Ilizarov. Chir Narz Ruchu Ortop Pol. 1994;LIX Supl:3236.

11. Lipsitz LA, Mukai S, Hamner J, et al. Dinamic regulation of middle cerebral artery blood flow velocity in aging and hypertension. Stroke. 2000;31(8):1897-1903.

12. Kulikov VP, Doronina NL, Fedjunina LG, et al. Ultrasound diagnosis of pathology of the arteries of the head and neck. In: The book: Ultrasound diagnosis of vascular diseases. Moscow: M. Firma Strom; 2007:122184.

13. Katz ML. Intracranial cerebrovascular evaluation. Textbook of Diagnostic ultrasonography. St Louis. Mosby-Year Book; 2001.

14. Olson Th P, Tracy J, Dengel DR. Relationship between ventilatory threshold and cerebral blood flow during maximal exercise in humans. The Open Sports Med Journ. 2009;3:9-13.

15. Zhelezkova AA, Skorobogatov Yu Yu, Filatova OV. Age-related changes in diameter of internal carotid arteries. News of Altai State University. 2010;3:26-29. 